\title{
A NOTE ON THE ABSOLUTE CONVERGENCE OF LACUNARY FOURIER SERIES
}

\author{
N. V. PATEL AND V. M. SHAH
}

\begin{abstract}
P. B. Kennedy [3] studied lacunary Fourier series whose generating functions are of bounded variation on a subinterval $I$ of $[-\pi, \pi]$ and satisfy a Lispschitz condition of order $\alpha$ on $I$. We show that the conclusion of one of his theorems on the absolute convergence of Fourier series remains valid when the function is merely of bounded $r$ th variation in $I$ and belongs to a class $\operatorname{Lip}(\alpha, p)$ in I. Our results also generalize three theorems of S. M. Mazhar [4].
\end{abstract}

\section{Introduction. Let}

$$
\sum_{k=1}^{\infty}\left(a_{n_{k}} \cos n_{k} x+b_{n_{k}} \sin n_{k} x\right)
$$

be the Fourier series of a $2 \pi$-periodic function $f \in L[-\pi, \pi]$, where $\left\{n_{k}\right\}(k \in N)$ is a strictly increasing sequence of natural numbers such that

$$
n_{k+1}-n_{k} \rightarrow \infty \quad \text { as } k \rightarrow \infty .
$$

P. B. Kennedy [3] proved the following theorem which recalls a well-known result of Zygmund [7, p. 136].

TheOrem A [3, TheOREM V(iv)]. If

(i) $\left\{n_{k}\right\}$ satisfies $(1.2)$,

(ii) $f$ is of bounded variation in a subinterval $I=\left[x_{0}-\delta, x_{0}+\delta\right]$ of $[-\pi, \pi]$, and

(iii) $f \in \operatorname{Lip} \alpha$ in $I$, where $0<\alpha<1$, then

$$
\sum_{k=1}^{\infty}\left(\left|a_{n_{k}}\right|+\left|b_{n_{k}}\right|\right)<\infty .
$$

In [4, Theorem 1] S. M. Mazhar generalized a theorem of Noble [5], obtaining a variant of Theorem A in which (i) is replaced by a stronger condition, while (ii) is replaced by the weaker condition that $f$ is of bounded $r$ th variation. In this paper we prove a generalization of both theorems. The classes of functions we consider are defined below.

Received by the editors September 20, 1983 and, in revised form, March 20, 1984

1980 Mathematics Subject Classification. Primary 42A28.

Key words and phrases. Lacunary Fourier series, absolute convergence, Lipschitz condition. bounded $r$ th variation.

1985 American Mathematical Society $0002-9939 / 85 \$ 1.00+\$ .25$ per page 
2. Definitions. (i) For $0<\alpha \leqslant 1$ and $p \geqslant 1, \operatorname{Lip}(\alpha, p, I)$ is defined to be the class of functions $f$ in $L[-\pi, \pi]$ such that

$$
\left(\int_{I}|f(x+h)-f(x)|^{p} d x\right)^{1 / p}=O\left(|h|^{\alpha}\right) \quad \text { as } h \rightarrow 0 .
$$

Evidently $\operatorname{Lip} \alpha \subseteq \operatorname{Lip}(\alpha, p)$ for every $p \geqslant 1$.

(ii) Suppose a function $f$ is defined on an interval $[a, b]$ where $a \leqslant x_{0}<x_{1}<\cdots$ $<x_{n-1}<x_{n} \leqslant b$. We write, as usual,

$$
\Delta^{1} f\left(x_{i}\right)=f\left(x_{i+1}\right)-f\left(x_{i}\right), \quad \Delta^{q} f\left(x_{i}\right)=\Delta^{q-1} f\left(x_{i+1}\right)-\Delta^{q-1} f\left(x_{i}\right) \quad(q \geqslant 2),
$$

so that

$$
\Delta^{q} f\left(x_{i}\right)=\sum_{\nu=0}^{q}(-1)^{\nu}\left(\begin{array}{l}
q \\
\nu
\end{array}\right) f\left(x_{i+q-\nu}\right) .
$$

Then $f$ is said to be of bounded $r$ th variation on $[a, b]$ (where $r$ is a positive integer) if there is a constant $M$ such that

$$
\sum_{i=0}^{n-r}\left|\Delta^{r} f\left(x_{i}\right)\right| \leqslant M
$$

whenever $x_{0}, x_{1}, \ldots, x_{n}$ are in arithmetical progression.

It is clear that every function of bounded variation is of bounded $r$ th variation, but the converse is false, as is pointed out by Mazhar [4].

We prove the following theorems.

\section{Results.}

THEOREM 1. If

(i) $\left\{n_{k}\right\}$ satisfies $(1.2)$,

(ii) $f$ is of bounded rth variation in $I$, and

(iii) $f \in \operatorname{Lip}(\alpha, p, I)$, where $0<\alpha \leqslant 1, p>2, \alpha p>1$, then (1.3) holds.

This theorem clearly generalizes the results of Kennedy and Mazhar referred to in $\S 1$. It may also be compared to a theorem of Hardy and Littlewood [2] in which $r=1, I=[-\pi, \pi]$, but lacunarity is not postulated.

The following extensions of Theorem 1 can also be proved; they contain Theorems 2 and 3 of [4].

THEOREM 2. If

(i) $\left\{n_{k}\right\}$ satisfies $(1.2)$,

(ii) f satisfies Theorem 1(ii), and

(iii) $f \in \operatorname{Lip}(\alpha, p, I)$, where $0<\alpha \leqslant 1, p>2$, then

$$
\sum_{k=1}^{\infty}\left(\left|a_{n_{k}}\right|^{\beta}+\left|b_{n_{k}}\right|^{\beta}\right)<\infty
$$

for every $\beta$ satisfying $2>\beta>2(p-1) /(2 p+\alpha p-3)$. 
THEOREM 3. Under the hypothesis of Theorem 2,

$$
\sum_{k=1}^{\infty} n_{k}^{\beta / 2}\left(\left|a_{n_{k}}\right|+\left|b_{n_{k}}\right|\right)<\infty
$$

for $\beta<(\alpha p-1) / 2(p-1)$.

Observe that, for $\beta=1$, Theorem 2 reduces to Theorem 1 , and for $\beta=0$, Theorem 3 also reduces to Theorem 1 .

4. Proof of Theorem 1. We need the following lemmas. Lemma 1 is a special case of a very general theorem due to Paley and Wiener [6, Theorem XLII']. Lemma 2 is due to Kennedy [3, Lemma 1]. The result of Lemma 3 is given in the introduction of the paper [1] by Hardy and Littlewood.

Lemma 1. Suppose the function $f \in L[-\pi, \pi]$ has Fourier series (1.1). If $n_{k+1}-n_{k}$ $\rightarrow \infty$ as $k \rightarrow \infty$ and $f \in L^{2}(I)$ for some subinterval $I$ of $[-\pi, \pi]$, then $f \in L^{2}[-\pi, \pi]$.

LEMMA 2. (i) Let $\left\{\lambda_{k}\right\}(-\infty<k<\infty)$ be a sequence of real numbers satisfying $\lambda_{k+1}-\lambda_{k} \rightarrow \infty(|k| \rightarrow \infty), \lambda_{k+1}-\lambda_{k}>8 \pi \delta^{-1}(\delta>0)$.

(ii) Let $\left\{A_{k}\right\} \quad(-\infty<k<\infty)$ be a sequence of complex numbers such that $\sum_{-\infty}^{\infty}\left|A_{k}\right| s^{\left|\lambda_{k}\right|}<\infty(0<s<1)$.

(iii) Let

$$
\phi(s, x)=\sum_{-\infty}^{\infty} A_{k} s^{\left|\lambda_{k}\right|} e^{i \lambda_{k} x} \quad(0<s<1,-\infty<x<\infty),
$$

(iv) Let

$$
\phi(x)=L^{2}-\lim _{s \rightarrow 1} \phi(s, x) \quad \text { in } I=\left[x_{0}-\delta, x_{0}+\delta\right]
$$

Then

$$
\sum_{-\infty}^{\infty}\left|A_{k}\right|^{2} \leqslant 8 \delta^{-1} \int_{I}|\phi(x)|^{2} d x
$$

Lemma 3. If $f \in \operatorname{Lip}(\alpha, p, I), p \geqslant 1$, then $f \in L^{p}(I)$.

Putting $n_{0}=0, n_{k}=-n_{-k}(k<0), C_{n_{0}}=0, C_{n_{k}}=\frac{1}{2}\left(a_{n_{k}}-i b_{n_{k}}\right)(k>0)$, and $C_{n_{k}}=\bar{C}_{n_{-k}}(k<0)$, we write $(1.1)$ in the form

$$
\sum_{k=-\infty}^{\infty} C_{n_{k}} e^{i n_{k} x}
$$

Since $C_{n_{k}} \rightarrow 0$ as $k \rightarrow \infty$,

$$
\sum_{k=-\infty}^{\infty}\left|C_{n_{k}}\right| s^{\left|n_{k}\right|}<\infty \quad(0<s<1)
$$

Put

$$
f(s, x)=\sum_{k=-\infty}^{\infty} C_{n_{k}} e^{i n_{k} x} s^{\left|n_{k}\right|} \quad(0<s<1,-\infty<x<\infty)
$$


Now, by Theorem 1(iii), $f \in \operatorname{Lip}(\alpha, 2, I)$ and, therefore, by Lemma $3, f \in L^{2}(I)$. Hence, by Lemma $1, f \in L^{2}[-\pi, \pi]$, and so, by a known theorem [7, p. 87], it follows that

$$
f(x)=L^{2}-\lim _{s \rightarrow 1} f(s, x) \quad(|x| \leqslant \pi) .
$$

Take integers $K, j$ such that

$$
n_{K}>2 r \pi / \delta \text { and } 0 \leqslant j \leqslant \delta n_{K} / 8 \pi .
$$

In the following argument we let $r$ be an even integer; the treatment for odd $r$ is similar.

Put

$$
g_{j}(x)=\sum_{\nu=0}^{r}(-1)^{\nu}\left(\begin{array}{l}
r \\
\nu
\end{array}\right) f\left(x+\frac{2 j \pi}{n_{K}}+\frac{(r-2 \nu) \pi}{2 n_{K}}\right)
$$

and

$$
A_{k j}=C_{n_{k}}\left(2 i \sin \frac{n_{k} \pi}{2 n_{K}}\right)^{r} \exp \left(\frac{i n_{k} 2 j \pi}{n_{K}}\right) .
$$

Since $\left|A_{k j}\right| \leqslant\left|C_{n_{k}}\right| 2^{r}$, it follows from (4.1) that

$$
\sum_{k=-\infty}^{\infty}\left|A_{k j}\right| s^{\left|n_{k}\right|}<\infty \quad(0<s<1) \text {. }
$$

If

$$
g_{j}(s, x)=\sum_{k=-\infty}^{\infty} A_{k j} s^{\left|n_{k}\right|} e^{i n_{k} x} \quad(0<s<1,-\infty<x<\infty),
$$

we have

$$
g_{j}(s, x)=\sum_{\nu=0}^{r}(-1)^{\nu}\left(\begin{array}{l}
r \\
\nu
\end{array}\right) f\left(s, x+\frac{2 j \pi}{n_{K}}+\frac{(r-2 \nu) \pi}{2 n_{K}}\right) ;
$$

from which, together with (4.2) and (4.4), we obtain

$$
g_{j}(x)=L^{2}-\lim _{s \rightarrow 1} g_{j}(s, x) \text { in }\left[x_{0}-\frac{1}{2} \delta, x_{0}+\frac{1}{2} \delta\right] \text {. }
$$

We may assume that

$$
n_{k+1}-n_{k}>16 \pi \delta^{-1} \text { for all } k .
$$

In view of (1.2), this can be achieved, if necessary, by adding to $f(x)$ a polynomial in $\exp \left(\operatorname{in}_{k} x\right)$, a process which affects neither the hypothesis nor the conclusion of the theorem. Hence, putting $I_{1}=\left[x_{0}-\frac{1}{2} \delta, x_{0}+\frac{1}{2} \delta\right]$, we obtain from (4.5)-(4.8) and Lemma 2 that

$$
\sum_{k=-\infty}^{\infty}\left|A_{k j}\right|^{2} \leqslant 16 \delta^{-1} \int_{I_{1}}\left|g_{j}(x)\right|^{2} d x
$$

But

$$
\left|A_{k j}\right|=\left|C_{n_{k}}\right| 2^{r}\left(\sin \frac{n_{k} \pi}{2 n_{K}}\right)^{r}
$$


Therefore (4.9) gives

$$
\sum_{n_{K} / 2}^{n_{K}} 2^{2 r}\left(\sin \frac{n_{k} \pi}{2 n_{K}}\right)^{2 r}\left|C_{n_{k}}\right|^{2} \leqslant 16 \delta^{-1} \int_{I_{1}}\left|g_{j}(x)\right|^{2} d x .
$$

But if $\frac{1}{2} n_{K} \leqslant\left|n_{k}\right| \leqslant n_{K}$, then

$$
\left(\sin \frac{\pi n_{k}}{2 n_{K}}\right)^{2 r} \geqslant\left(\frac{1}{2}\right)^{r}
$$

Therefore $(4.10)$ reduces to

$$
\sum_{n_{K} / 2}^{n_{K}}\left|C_{n_{k}}\right|^{2} \leqslant \frac{16 \delta^{-1}}{2^{r}} \int_{I_{1}}\left|g_{j}(x)\right|^{2} d x .
$$

Let $p^{\prime}=p-1$. Since $p>2, p^{\prime}>1$. Define $q$ by the equation $1 / p^{\prime}+1 / q=1$.

We now denote, by $\sum_{j}$, summation over the range $0 \leqslant j \leqslant\left[\delta n_{k} / 8 \pi\right]$ (where $[a]$ represents the integral part of $a$ ). It then follows from (4.11) that

$$
\begin{aligned}
\left\{\sum_{n_{K} / 2}^{n_{K}}\left|C_{n_{k}}\right|^{2}\right\}^{q} & \leqslant \frac{\left(16 \delta^{-1} 2^{-r}\right)^{q}}{\left[\delta n_{K} / 8 \pi\right]} \sum_{j}\left\{\int_{I_{1}}\left|g_{j}(x)\right|^{2} d x\right\}^{q} \\
& \leqslant \frac{C}{n_{K}} \sum_{j}\left\{\int_{I_{1}}\left|g_{j}(x)\right|^{2} d x\right\}^{q}
\end{aligned}
$$

where the constant $C$ depends on $\delta$ and $r$. $C$ will henceforth denote positive constants not necessarily the same at each occurrence.

Since $2=\left(p^{\prime}+1\right) / p^{\prime}+1 / q$, an application of Hölder's inequality gives

$$
\begin{aligned}
\left\{\int_{I_{1}}\left|g_{j}(x)\right|^{2} d x\right\}^{q} & =\left\{\int_{I_{1}}\left|g_{j}(x)\right|^{\left(p^{\prime}+1\right) / p^{\prime}+1 / q} d x\right\}^{q} \\
& \leqslant\left[\left(\int_{I_{1}}\left|g_{j}(x)\right|^{\left(p^{\prime}+1\right) p^{\prime} / p^{\prime}} d x\right)^{1 / p^{\prime}}\left(\int_{I_{1}}\left|g_{j}(x)\right|^{1 / q \cdot q} d x\right)^{1 / q}\right]^{q} \\
& =\left\{\int_{I_{1}}\left|g_{j}(x)\right|^{p} d x\right\}^{q / p^{\prime}}\left\{\int_{I_{1}}\left|g_{j}(x)\right| d x\right\} .
\end{aligned}
$$

This, combined with (4.12), gives

$$
\left\{\sum_{n_{K} / 2}^{n_{K}}\left|C_{n_{k}}\right|^{2}\right\}^{q} \leqslant \frac{C}{n_{K}} \sum_{j}\left\{\left(\int_{I_{1}}\left|g_{j}(x)\right|^{p} d x\right)^{q / p^{\prime}} \int_{I_{1}}\left|g_{j}(x)\right| d x\right\} .
$$

Putting $y=x+2 j \pi / n_{K}+r \pi / 2 n_{K}$ in (4.4), we evidently have

$$
g_{j}(x)=\sum_{\nu=0}^{r-1} a_{\nu}\left\{f\left(y-\frac{\nu \pi}{n_{K}}\right)-f\left(y-\frac{(\nu+1) \pi}{n_{K}}\right)\right\},
$$

where $a_{0}, a_{1}, \ldots, a_{r-1}$ are constants. Hence, by Minkowski's inequality and condition Theorem 1(iii),

$$
\begin{aligned}
{\left[\int_{I_{1}}\left|g_{j}(x)\right|^{p} d x\right]^{1 / p} } & \leqslant \sum_{\nu=0}^{r-1}\left|a_{\nu}\right|\left\{\int_{I}\left|f\left(y-\frac{\nu \pi}{n_{K}}\right)-f\left(y-\frac{(\nu+1) \pi}{n_{K}}\right)\right|^{p} d x\right\}^{1 / p} \\
& =O\left(n_{K}^{-\alpha}\right) .
\end{aligned}
$$


Also, for $\left|x-x_{0}\right| \leqslant \delta / 2$ and integers $j$ and $K$ satisfying (4.3), the points $y-\nu \pi / n_{K}$ $(\nu=0,1, \ldots, r)$ all lie in $\left[x_{0}-\delta, x_{0}+\delta\right]$. Therefore, if $V$ is the total $r$ th variation of $f$ in $I$, it follows from Theorem 1(ii) that

$$
\sum_{j}\left|g_{j}(x)\right| \leqslant V \quad \text { for }\left|x-x_{0}\right| \leqslant \frac{1}{2} \delta .
$$

Hence, by (4.13),

$$
\left\{\sum_{n_{K} / 2}^{n_{K}}\left|C_{n_{k}}\right|^{2}\right\}^{q} \leqslant C n_{K}^{-1} n_{K}^{-\alpha p q / p^{\prime}} V \delta=C n_{K}^{-1-\alpha p q / p^{\prime}},
$$

which in turn gives

$$
\sum_{n_{K} / 2}^{n_{K}}\left|C_{n_{k}}\right|^{2} \leqslant C n_{K}^{-1 / q-\alpha p / p^{\prime}} .
$$

Let $m$ be a positive integer. Either the set of integers $k$ for which $2^{m}<\left|n_{k}\right| \leqslant 2^{m+1}$ is empty, or there is a member of this set, say $K=K(m)$, which has largest modulus; and in the latter case, the set is included in the set of $k$ for which $\frac{1}{2} n_{K} \leqslant\left|n_{k}\right| \leqslant n_{K}$. Moreover, if $m$ is large enough, $K$ satisfies (4.3). Hence, in either case it follows from (4.14) that

$$
\begin{aligned}
\sum_{2^{m}<\left|n_{k}\right| \leqslant 2^{m+1}}\left\{\left|C_{n_{k}}\right|^{2}\right\} & \leqslant \sum_{n_{K} / 2 \leqslant\left|n_{k}\right| \leqslant n_{K}}\left\{\left|C_{n_{k}}\right|^{2}\right\}=O\left(n_{K}^{-1 / q-\alpha p / p^{\prime}}\right) \\
& =O\left(2^{m\left(-1 / q-\alpha p / p^{\prime}\right)}\right) .
\end{aligned}
$$

Also, by (4.8), the number of terms in the above summation is $O\left(2^{m}\right)$. Hence, by Cauchy's inequality,

$$
\begin{aligned}
\sum_{2^{m}<\left|n_{k}\right| \leqslant 2^{m+1}}\left|C_{n_{k}}\right| \leqslant & \left\{\sum_{2^{m}<\left|n_{k}\right| \leqslant 2^{m+1}}\left|C_{n_{k}}\right|^{2}\right\}^{1 / 2}\left\{\sum_{2^{m}<\left|n_{k}\right| \leqslant 2^{m+1}} 1\right\}^{1 / 2} \\
& =O\left\{2^{m / 2\left((1-\alpha p) / p^{\prime}\right)}\right\},
\end{aligned}
$$

so

$$
\sum_{k=-\infty}^{\infty}\left|C_{n_{k}}\right| \leqslant C \sum_{m=1}^{\infty} 2^{m(1-\alpha p) / 2 p^{\prime}} .
$$

Since $\alpha p>1$, the series on the right side is convergent. This completes the proof of Theorem 1.

Theorems 2 and 3 can be proved in a similar manner.

Thanks are due to the referee for his useful comments.

\section{REFERENCES}

1. G. H. Hardy and J. E. Littlewood, Some properties of fractional integrals. I, Math. Z. 27 (1928), $565-606$.

2. N__ Notes on the theory of series. IX: On the absolute convergence of Fourier series, J. London Math. Soc. 3 (1928), 250-253.

3. P. B. Kennedy, Fourier series with gaps, Quart. J. Math. Oxford Ser. (2) 7 (1956), 224-230. 
4. S. M. Mazhar, On the absolute convergence of a Fourier series with gaps, Proc. Nat. Acad. Sci. India Sect. A 26 (1960), 104-109.

5. M. E. Noble, Coefficient properties of Fourier series with a gap condition, Math. Ann. 128 (1954), $55-62$.

6. R. E. A. C. Paley and N. Wiener, Fourier transforms in the complex domain, Amer. Math. Soc. Colloq. Publ., Vol. 19, Amer. Math. Soc., Providence, R. I., 1934.

7. A. Zygmund, Trigonometrical series, PWN, Warsaw, 1935.

Department of Mathematics, Faculty of Science, The Maharaja Sayajirao University of BARODA, BARODA - 390 002, INDIA 